Apidologie, 1984, 15 (3), 339-354

\title{
REGULIERUNG DES PROTEINHAUSHALTES IM BIENENVOLK (APIS MELLIFICA L.) DURCH BRUTKANNIBALISMUS
}

\author{
K. WEISS \\ Bayerische Landesanstalt für Bienenzucht \\ Burgbergstraße 70, 8520 Erlangen
}

ZUSAMMENFASSUNG

Jungbienen können anstelle von Pollen Bienenbrut verzehren und erreichen damit denselben Effekt wie beim Pollenfraß : sie werden langlebig und fähig, Brut aufzuziehen. Dadurch ist das Volk in der Lage, kritische Umweltsituationen leichter zu überwinden.

Man kann in der praktischen Bienenzucht nicht selten beobachten, daß Bienenbrut aus Bienenvölkern verschwindet, ohne zur Deckelung, geschweige denn zum Schlupf zu gelangen. Oft kommt die Brut nicht einmal über das Eistadium hinaus. So kann es passieren, daß man im Frühjahr eine Drohnenwabe bei späterer Nachschau wieder leer findet. Dafür gibt es zwei mögliche Ursachen : Entweder ziehen sich die Bienen bei kühlem Wetter aus den Randzonen des Volkes zurück, so daß die dort angelegte Drohnenbrut verkühlt, oder die Drohnenbrut entwickelt sich nicht, weil es im Volk an Pollen mangelt. Eine Bestätigung der letztgenannten Möglichkeit darf man darin sehen, daß sich dem Eiverlust durch Zufuhr von Pollen oder Pollenersatz vorbeugen läßt. Das erreicht man, wenn man das Eiweißfutter in den oberen Teil der Drohnenwabe einfüllt und mit Honigwasser übersprüht oder es in Form eines hochprozentigen Eiweißteiges über die Drohnenwabe legt (WEiss, 1958). Eier und junge Brut verschwinden manchmal auch aus Arbeiterwaben - vermutlich aus denselben Gründen. MYSER (1952) und Fukuda und SaKagami (1960) beobachteten das Verschwinden von Eiern in Schaukästen, welche wärmetechnisch immer problematisch sind. Nach Feststellungen von MERz (1979) waren es allerdings nicht Eier, sondern die daraus geschlüpften Maden, die dort im zeitigen Frühjahr regelmäßig aus den Zellen verschwanden.

Die Aufzucht von Brut ist in der Regel auf das Vorhandensein von Polleneiweiß angewiesen. Stoppt in Schlechtwetterzeiten die Pollenversorgung, kann es 
zur Beseitigung der Brut in den Völkern kommen. Immer ist dabei zuerst die Drohnenbrut gefährdet. Abgesehen vom herbstlichen Drohnenabtrieb, wobei auch häufig Drohnenbrut mit hinausgeworfen wird, kann man beides gelegentlich auch mitten im Sommer beobachten, wenn längere Schlechtwetterzeiten die Aktivität der Bienenvölker einschränken.

Zählende und messende Untersuchungen zur Überlebensfähigkeit der Brut in den Bienenvölkern lassen den Schluß zu, daß eine gewisse Ausfallquote eher Regel als Ausnahme ist. Merrill (1924) fand zwischen der Zahl der abgesetzten Eier einer Königin und der zu erwartenden gedeckelten Brut ein permanentes Minus, dessen Umfang im Laufe des Jahres variierte. Unterschiedliche tägliche Überlebensraten bei den verschiedenen heranwachsenden Brutstadien konstatierte Garófalo (1972), wobei große Unterschiede zwischen den Völkern zu verzeichnen waren. Folgerungen ähnlicher Art lassen sich auch aus den Untersuchungen zum Massenwechsel des Bienenvolkes von WILLE und GerIG (1976) ziehen. WoYke (1977) stellte bei regelmäßigen Nachschauen in Völkern von Apis mellifica fest, daß in erster Linie die jüngste (1-3 tägige), weniger die ältere Brut verschwand. Dabei unterlag der Brutverlust offenbar einem jährlichen Rhythmus. Im Frühjahr verschwanden 20-25 \%, im Sommer 10-20\% und im Herbst $25-50 \%$ der Brut. Auch gab es aufschlußreiche Unterschiede zwischen dem Verschwinden von Drohnen- und Arbeiterbrut in Bezug auf Jahreszeit und Weiselrichtigkeit. Besonders große Ausfälle an heranwachsender Brut hat WoYke (1976) bei Völkern der Indischen Honigbiene (Apis cerana) in winterfreien Zonen konstatiert, wobei vor allem auch ältere Larven verschwanden. WOYKE stellte einen engen Zusammenhang zwischen dem Brutausfall und der Ergiebigkeit der Nektar- und Pollentracht fest. Nach seinen Beobachtungen können aber auch Störungen anderer Art, z.B. Platzveränderung einer Brutwabe, Brutverluste auslösen. Bei Apis cerana hat schon die Inspektion einer Drohnenwabe, die an ihren angestammten Platz zurückgehängt wurde, zum Ausräumen einiger oder aller Brut durch die Bienen geführt. MonTAGNER (1962) fand beim Einhängen von Waben mit Eiern oder jungen Maden in Bienenvölker, daß die Weiterpflege der Brut außer vom Zustand des Volkes (wahrscheinlich Zahl und Beschaffenheit der vorhandenen Ammen) vor allem vom Vorhandensein gedeckelter Brut und der Entfernung der zuzuhängenden Wabe von der Königin abhing. Gröbere Eingriffe, wie die manuelle Öffnung von bereits gedeckelten Brutzellen in einem Bienenvolk (Nauleau, 1960 und Newton und Michl, 1974) führten zu unterschiedlichen Brutverlusten, bei denen die Eigenart des Volkes, die Zeit des Eingriffs, der Umfang der entdeckelten Wabenflächen, das Alter des Zellinhaltes und die Versorgung des Volkes mit Pollen eine Rolle spielten.

Von besonderer Bedeutung ist die Frage nach dem Verbleib der verschwundenen Brut. WoyKe (1977) suchte vergeblich auf dem Bodenbrett nach abgefallenen Brutstadien und es gelang ihm nicht, das Heraustragen von Brut 
aus dem Flugloch zu beobachten. Auch Nauleau (1960) suchte erfolglos nach Spuren von verschwundenen Larven und Vorpuppen. Die Autoren schlossen daraus, daß die Bienen die Brut gefressen haben mußten. NEwTon und Michl (1954) und WOYKE (1976) konnten aber auch Bienen direkt beim Brutfraß beobachten. WOYKE fand zudem angefressene Larven, die sich noch bewegten.

Der Brutfraß bei den Bienen ist nichts Ungewöhnliches und auch in anderem Zusammenhang bekannt. So erkennen die Bienen nach Woykt: (1963) die diploide Beschaffenheit der jüngsten Drohnenmaden in ingezüchteten Völkern und fressen sie auf. Es ist anzunehmen, daß infizierte Brut (Europäische oder Amerikanische Faulbrut) von den Bienen ebenfalls verzehrt wird (ScHULZLANGNER, 1956). Wenn es auch nicht ganz leicht ist, das Schicksal verschwindender Eier und Larven unter natürlichen Bedingungen unmittelbar zu verfolgen, so gibt es für ältere, bereits gedeckelte Brut mitunter doch recht eindeutige Hinweise. Verletzt man bei der Bearbeitung eines Bienenvolkes gelegentlich Brutbezirke mit Streckmaden oder Vorpuppen, stürzen sich die Bienen in der Regel auf die beschädigten Brutstadien und saugen die auslaufende «Brutmilch» auf. Durch das «Köpfen» der Drohnenbrut hat man dieses Verhalten früher geradezu herausgefordert, weil man glaubte, daß die Völker mit reduziertem Drohnenbestand leistungsfähiger seien. Obgleich diese Ansicht heute widerlegt ist (Lit. bei WeIss, 1969), dürfen wir auf der anderen Seite auch die verschiedentlich geäußerte antroposophische Vorstellung, daß der Fraß der Drohnenbrut die Bienen «demoralisieren» und zur Tatenlosigkeit verurteilen könnte, als ein Märchen abtun.

So sicher wir annehmen dürfen, daß die Bienen beschädigte, infizierte oder verkühlte Brut mitunter gänzlich aus dem Volk entfernen (die Imkerpraxis gibt dafür genügend Beispiele!), so gewiß ist es auch, daß sie unter bestimmten Umständen Brut jeden Alters aufzehren. Die auffällige Abhängigkeit des Brutfraßes von den herrschenden Trachtbedingungen deutet darauf hin, daß der Eiweißbedarf dabei eine starke, vielleicht die stärkste, Triebfeder darstellt. WoYkE (1976) spricht bereits die Vermutung aus, daß die Bienen Brut fressen, um Futtersaft zu produzieren, mit welchem sie die restliche Brut versorgen.

Diese Annahme schien mir eines experimentellen Beleges wert. Ich fragte also : Können die Bienen mit der tierischen Eiweißnahrung, als welche die Brut anzusehen ist, etwas anfangen? Vertragen sie sie und nützen sie sie? Ich habe zur Beantwortung dieses Sachverhaltes eine Reihe von Laborversuchen durchgeführt. 


\section{VERZEHREN GEKÄFIGTE BIENEN BRUT UNI WIE BEKOMMT SIE IHNEN?}

\section{A. Versuchstechnik}

Ich verwendete zu den Versuchen Holzkästchen mit den Innenmaß3en $9 \times 9 \times 4 \mathrm{~cm}$. Sie waren auf der Vorderseite mit einer hochschiebbaren Glasscheibe, auf der Rückseite mit einer Gitterwand versehen. In jedes Kästchen brachte ich 50 cben im Brutschrank geschlüpfte Bienen. Die Futterversorgung mit Zuckerwasser $(1: 1)$ erfolgte aus Futtergläschen mit Schraubverschluß, welche durch ein oberes Loch in den Versuchskäfig eingeführt und mit einem Gummiring festgehalten wurden. Außerdem bot ich den Bienen in einem Schälchen frische, eben aus der Wabe entfernte Bienenbrut. In jedem Kästchen wurde nur jeweils ein bestimmtes Brutalter gereicht : 2,5-3, 4 und 5 Tage alte Larven, Vorpuppen und Puppen. Von den 2,5-3 tägigen Maden gab ich täglich 10-30, von den übrigen Stadien 6 Tiere. Über das Wochenende wurden die Rationen verdoppelt.

In gleicher Weise mit Zuckerwasser und Bienenbrut versorgte ich eine Reihe von Kontrollkästchen, in denen die Bienen zusätzlich Pollen bekamen, der frisch aus den Waben entnommen und ebenfalls in Schälchen angeboten wurde. In einem Kästchen erhielten die Bienen nur Zuckerwasser und Pollen, und in einem weiteren Zuckerwasser allein. Die Versuchskäfige standen während der ganzen Versuchsdauer im Brutschrank bei $30^{\circ} \mathrm{C}$ und ca. $60 \%$ r.F. Sie wurden nur während der Fütterung herausgenommen.

\section{B. Versuchsverlauf und Ergebnisse}

Die gekäfigten Bienen begannen in der Regel an ihrem 3. Lebenstag mit dem Verzehr der im Näpfchen gebotenen Brut und - soweit gereicht - des Pollens. Ausnahmsweise dauerte es auch 6 Tage, was möglicherweise mit dem Alter der Futtermaden zusammenhing. Von der verzehrten Brut blieb in der Regel nichts oder nur ein Rest vertrockneter Häute übrig. Letzteres traf besonders für Vorpuppen und Puppen zu.

Wie es nach den Ergebnissen früherer Käfigversuche (Böttcher, Hirschfelder, Weiss, 1963) nicht anders zu erwarten war, nahmen die Bienen nach ihrem 12. Lebenstag so gut wie keinen Pollen mehr zu sich. Sie fraßen aber in der Regel wenigstens bis zu ihrem 15. Lebenstag Brut und auch danach hielt der Brutverzehr in verringertem Umfang noch eine Reihe von Tagen an (geklammerte Zahlen in Spalte 2 der Tabelle 1). Die Maden, Vorpuppen und Puppen wurden dann nur noch angefressen, der Rest verfärbte sich schwarz und trocknete ein. Die Bienen zernagten die Körperhülle oft in kleine Teilchen. Ich entfernte jeweils alles, bevor ich neues Brutfutter bot.

Die brutverzehrenden Bienen machten während dieser Zeit einen besonders nervösen und angriffslustigen Eindruck. Man hatte beim Wechsel der Futterschälchen Mühe, die Bienen nicht aus den Versuchskästchen entkommen zu lassen. Auch war der Verbrauch an Zuckerwasser während der Brutfütterung sehr hoch. Normalerweise reicht bei 50 gekäfigten Bienen ein Futtergläschen mit $6 \mathrm{ml}$ Zuckerwasser von Montag bis Freitag, bei unserem Versuch mußte indessen regelmäßig zwischengefüttert werden. Oft war zu beobachten, daß die Bienen Larven und besonders Vorpuppen und Puppen im Käfig umherschleppten und offenbar daraus $z u$ entfernen versuchten. Vielleicht hängt der erhöhte Futterverbrauch mit dem durch diese Aktivitäten bedingten erhöhten Kraftaufwand zusammen. Sobald allerdings die Bienen den Brutfraß eingestellt hatten, verhielten sie sich wieder normal. Sie wurden ruhiger und der Zuckerwasserverbrauch ging zurück.

Aus Spalte 4 in der Tabelle 1 wird ersichtlich, daß sowohl die ohne Pollen gehaltenen, als auch die mit Pollenzusatznahrung versorgten Bienen Brut verzehren. Die gefressene Brutmenge war bei den «Pollenbienen» etwas geringer. Bei der Ermittlung der Anzahl gefressener Tiere habe ich die nur teilweise verzehrten Larven und Puppen anteilsmäßig aufgerechnet. Die Gewichte wurden durch Wiegen (vor der Fütterung) und Schätzen (unter Berücksichtigung der nicht verzehrten Brut während und am Ende des Brutfraßes) ermittelt. 
TAB. 1. - Versuch über die Lebensdauer brutfressender Bienen

TABL. 1. - Experiment on the life span of brood eating bees

\begin{tabular}{|c|c|c|c|c|}
\hline 1 & 2 & 3 & 4 & 5 \\
\hline $\begin{array}{l}\text { Vers. Nr. } \\
\text { Exper. } \\
\text { no. }\end{array}$ & $\begin{array}{c}\text { Alter der Bienen } \\
\text { zu Beginn/am Ende } \\
\text { d. Brutfraßes in Tagen } \\
\text { Age of bees at the } \\
\text { beginning/end of } \\
\text { broodeating in days }\end{array}$ & $\begin{array}{c}\text { Alter der verfütterten } \\
\text { Tiere } \\
\text { Age of the eaten brood }\end{array}$ & $\begin{array}{l}\text { Zahl und Gewicht }(\mathrm{g}) \\
\text { der gefressenen Tiere } \\
\text { Number } \\
\text { and weight (g) } \\
\text { of the eaten brood }\end{array}$ & $\begin{array}{l}\text { Halbwertszeit } \\
\text { der Lebensdauer } \\
\text { der Bienen } \\
\text { in Tagen } \\
\text { Day to } 50 \% \\
\text { mortality }\end{array}$ \\
\hline
\end{tabular}

Versuche mit Pollen-Zufutter

Trials with additional pollen food

\begin{tabular}{|c|c|c|c|c|c|c|}
\hline 1 & 6 & $15(25)$ & $\begin{array}{l}\text { Larven ( } 3 \text { Tage) } \\
\text { Larvae ( } 3 \text { days) }\end{array}$ & 115 & $(2,30)$ & 42 \\
\hline 2 & 3 & $15(25)$ & $\begin{array}{l}\text { Larven ( } 4 \text { Tage) } \\
\text { Larvae (4 days) }\end{array}$ & 82 & $(5,70)$ & 43 \\
\hline $3 a$ & 3 & $15(23)$ & $\begin{array}{l}\text { Larven ( } 5 \text { Tage) } \\
\text { Larvae ( } 5 \text { days) }\end{array}$ & 65 & $(10,40)$ & 46 \\
\hline b & 3 & $7(21)$ & $\begin{array}{l}\text { Larven ( } 5 \text { Tage) } \\
\text { Larvae ( } 5 \text { days) }\end{array}$ & 43 & $(6,90)$ & 43 \\
\hline 4 & 2 & $7(20)$ & $\begin{array}{l}\text { Vorpuppen } \\
\text { Prepupae }\end{array}$ & 41 & $(6,60)$ & 36 \\
\hline 5 & 3 & $15(24)$ & $\begin{array}{l}\text { Puppen } \\
\text { Pupae }\end{array}$ & 58 & $(8,70)$ & 40 \\
\hline 6 & - & - & - & - & - & 40 \\
\hline
\end{tabular}

Versuche ohne Pollen-Zufutter

Trials without additional pollen food

\begin{tabular}{|c|c|c|c|}
\hline 7 & 6 & $15(25)$ & $\begin{array}{l}\text { Larven ( } 3 \text { Tage) } \\
\text { Larvae (3 days) }\end{array}$ \\
\hline 8 & 3 & $15(25)$ & $\begin{array}{l}\text { Larven (4 Tage) } \\
\text { Larvae (4 days) }\end{array}$ \\
\hline $9 \mathrm{a}$ & 3 & $15(23)$ & $\begin{array}{l}\text { Larven ( } 5 \text { Tage) } \\
\text { Larvae (5 days) }\end{array}$ \\
\hline b & 3 & $15(21)$ & $\begin{array}{l}\text { Larven (5 Tage) } \\
\text { Larvae (5 days) }\end{array}$ \\
\hline $10 a$ & 3 & $15(24)$ & $\begin{array}{l}\text { Vorpuppen } \\
\text { Prepupae }\end{array}$ \\
\hline b & 2 & $8(20)$ & $\begin{array}{l}\text { Vorpuppen } \\
\text { Prepuae }\end{array}$ \\
\hline $11 \mathrm{a}$ & 3 & $15(24)$ & $\begin{array}{l}\text { Puppen } \\
\text { Pupae }\end{array}$ \\
\hline b & 2 & $7(16)$ & $\begin{array}{l}\text { Puppen } \\
\text { Pupae }\end{array}$ \\
\hline 12 & - & - & \\
\hline
\end{tabular}

\begin{tabular}{lc|l}
128 & $(2,60)$ & 26 \\
91 & $(6,35)$ & 30 \\
73 & $(11,70)$ & 46 \\
70 & $(10,60)$ & 40 \\
65 & $(10,40)$ & 40 \\
53 & $(8,50)$ & 33 \\
72 & $(10,00)$ & 44 \\
41 & $(6,15)$ & 37 \\
- & - & 20
\end{tabular}


Bei der Feststellung der Icbenserwartung der gekäfigten Bienen in Spalte 5 ist die Zeit in Tagen angegeben, nach der genau die Hälfte der Bienen gestorben war. Fs zeigte sich :

1. Mit 5tägigen Larven Vorpuppen und Puppen gefütterte Bienen lebten mit und ohne Pollenzusatzfutter etwa gleich lang (Vers. Nr. 3-5 u. 9-11). Die Halbwertszeit ihrer Lebensdauer lag zwischen 36 und 46 bzw. 33 und 46 Tagen. Sie lebten somit etwa ebenso lang wie die Kontrollbienen, dic keine Brut, aber Pollen, bekamen (Vers. Nr. 6).

2. Etwa die gleiche Lebensdauer erreichten Versuchsbienen, welche mit jüngerer Brut $(2$ 1/2-3 und 4tägigen Maden) und zusätzlich mit Pollen gefüttert wurden (Vers. Nr. 1 u. 2). Wenn die Bienen aber zusätzlich zu diesen jungen Brutstadien keinen Pollen bekamen, lebten sie kürzer, nur 26 bzw. 30 Tage (Vers. Nr. 7 u. 8).

3. Die Kontrollen ohnc Brut und Pollen lebten am kürzesten. Die Halbwertszeit der Lebensdauer betrug hier nur 20 Tage (Vers. Ni. 12).

\section{KÖNNEN DIE BIENEN DIE VERZEHRTE BRIT ZUR BRUTPFLEGE NUTZEN?}

\section{A. Versuchstechnik}

Verwendet wurden dicselben Versuchskifige wic bei der ersten Fragestellung, und anch die Besetzung mit Bienen war dic gleiche. Die Bienen erhielten wieder Zuckerwasser 1 : 1 ad libitum. In einer ersten Versuchsreihe gab ich anstatt Maden in Schälchen ausschließ3lich kleine Wabenstücke mit Bienenbrut. Dabei legte ich Wert darauf, daß die Maden einerseits nicht zu jung waren, damit sic noch einen lohnenden Nahrungsfaklor abgaben, andererseits sollten sie aber aluch noch eine gewisse Fütterungs- und Entwicklungszeil vor sich haben. So verwendete ich vorwiegend 212.3 Tage alte Maden, die noch 3 Tage Futter e:halten mußten, che sie gedeckelt wurden.

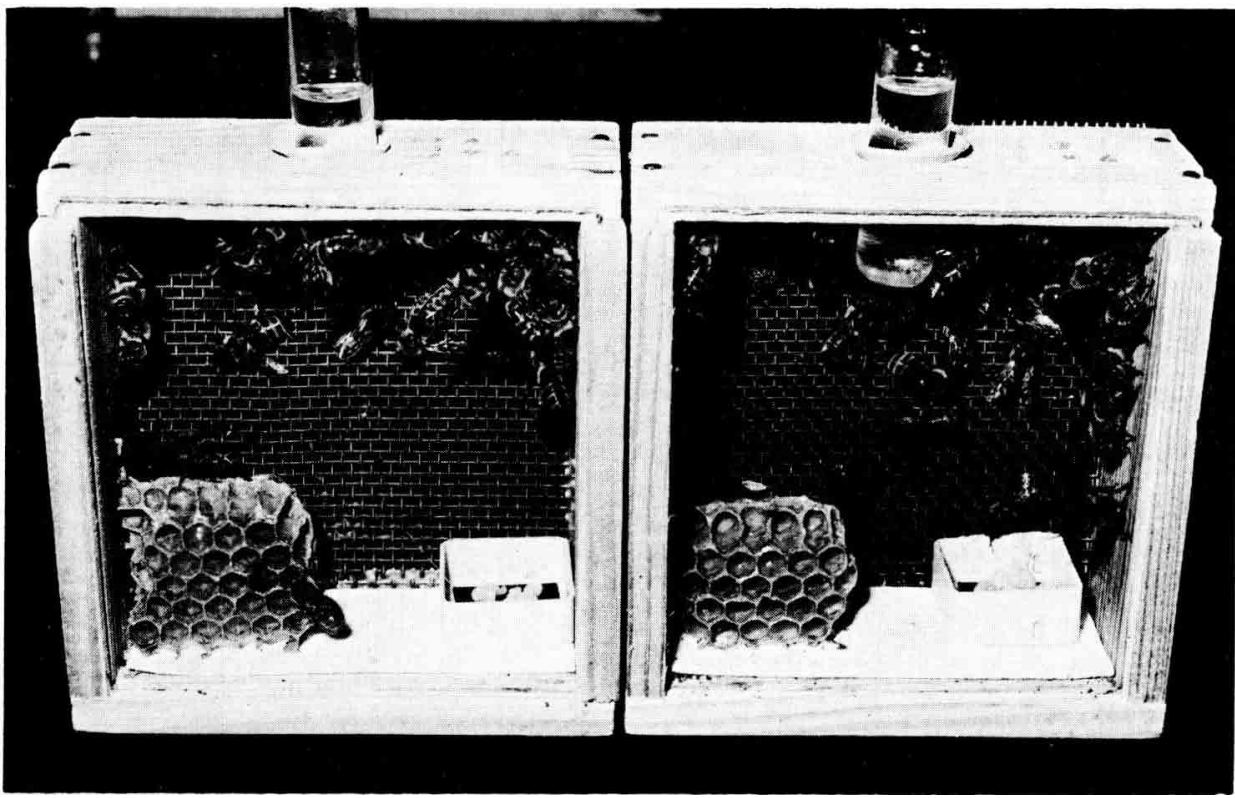

Are. 1. - Versuchskäfige mit Bienen, Brutwabenstiicken und Schälchen für Brut als Zusatzahlurang

Fri. 1. - Cages with bees, pieces of combs, and dishes with larae as additional food 
Die Wabenstücke enthielten jeweils etwa 10-30 bebrütete Zellen. Anfangs gab ich immer dann ein neues Wabenstïck mit frischen Larven, wenn die Bienen das vorhergehende leergefressen oder gedeckelt hatten. Das bedeutete in der Regel mehrmaligen Wabenwechsel in der Woche. Später genügte es, die Wabe 1 mal wöchentlich zu wechseln.

Die Wabenstücke brachtc ich zuerst in natïrlicher Stellung, also mit seitwärts ausgerichteten Zellen, in die Versuchskästchen. Es zeigte sich aber, daß man die Waben auch mit den Zellen nach oben auf den Boden legen konnte, ohne den Versuch dadurch zu beeintiächtigen. Die Maden auf der Unterseite wurden in diesem Fall vorher entfernt.

In einer zweiten Versuchsreihe elhielten die Bienen neben dem Brutwabenstück, in dem sich wieder 2 1/2-3 Tage alte Larven befanden, zusätzlich Brutnahrung aus einem Schälchen. Ich verwendete entweder 2 1/2-3 Tage alte Larven, wie im Wabenstück, oder Streckmaden bzw. Vorpuppen, die ich kurz vorher aus Brutwaben entnahm. Außerdem bemühte ich mich, die täglichen Futterrationen gleich zu halten, indem ich die zu fütternde Brut vorher auswog. Ich reichte entweder die konstante Zahl von 5 Vorpuppen oder gab so viele 2 1/2-3 tätige Larven, wie dem Gewicht der 5 Vorpuppen (680-690 mg) entsprachen. Das konnten je nach Größe 30-50 Iarven sein. Solange die Bienen das gebotene Brutfutter vollständig aufnahmen, war das Gewicht exakt zu bestimmen. Der Schätzung bedurfte indessen das Gewicht der Larven in den Wabenzellen, soweit die Brut von den Bienen gefressen und nicht gepflegt wurde. Dabei halfen gelegentlich durchgefüh:te Probewägungen. Wenn die Bienen die Larven in den Schälchen nur noch teilweise auffraßen, war eine Schätzung des Gewichtes der verzehrten Brut ebenfalls angebracht, da je nach dem Alter der Larven die überbleibenden Reste verschieden stark austrockneten, so daß deren Rückwägung nicht sinnvoll gewesen wäre.

\section{B. Versuchsergebnisse (Tab. 2)}

Grundsätzlich ist festzustellen, daß die pollenlos aufgezogenen Käfigbienen in der Lage waren, Brut zu pflegen. Sie verschafften sich die nötigen Aufbaustoffe durch den Verzehr von Brut. Im einzelnen zeigte sich :

1. Wenn die Bienen zur Brutaufzucht auf Brut in den Wabenstücken angewiesen waren (Vers. Nr. 1-3), nahmen sie a) bedeutend weniger Brutzellen in Pflege und begannen b) später mit der Brutpflege als wenn sie zusätzlich Pollen erhielten (Vers. Nr. 4-6). Ohne Pollen fraßen die Bienen in den Versuchen $\mathrm{Nr} .1$ u. 2 die ersten 5 nacheinander gereichten Wabenstücke vollkommen leer, ehe 18 Tage nach dem Versuchsansatz auf dem 6. Wabenstück je 1 Zelle gedeckelt wurde. In Vers. Nr. 2 deckelten sie auf dem 7. Wabenstück noch einmal 2 (insgesamt 3) Zellen und in Versuch Nr. 3 (mit älteren Maden !), bei dem es schon nach 9 Versuchstagen auf dem 3. Wabenstïck zur ersten Deckelung kam, erfolgten nacheinander 3 Deckelungen mit 1,10 und 4 (insgesamt 15) Zellen.

Mit Pollenzusatzfutter traten in den Versuchen 4 und 5 die ersten gedeckelten Zellen 11 Tage nach dem Versuchsansatz auf, nachdem die Bienen die beiden ersten Wabenstücke leergefressen hatten. Sic pflegten auf 2 aufeinanderfolgenden Wabenstücken insgesamt je 13 Zellen. In Vers. $\mathrm{Nr}$. 6 (mit älteren Maden!) erschienen die ersten gedeckelten Zellen bereits. auf dem ersten gebotenen Wabenstück nach 6 Tagen, und es wurden auf 3 aufeinanderfolgenden Wabenstücken insgesamt 21 Zellen gedeckelt.

Der Deckelungserfolg war erwartungsgemäß bei diesen beiden Versuchsgruppen unter Verwendung von Wabenstücken mit älteren, also schwereren und schneller deckelungsreifen Maden größer als bei den Stücken mit jungen Maden.

2. Wenn die Bienen anstelle von Pollen Bienenbrut ad libitum zugeführt bekamen, zogen sie noch mehr Brut auf, auch wenn die Larven in den Wabenstücken nur 2 1/2-3 Tage alt waren (Vers. Nr. 7-10). Ob der Unterschied in der Zahl der gedeckelten Zellen (49 und 45 Zellen in Vers. Nr. 7 u. 8 gegen 25 und 35 in Vers. Nr. 9 u. 10) mit dem zugefütterten Larvenalter (2 1/2-3 Tage bzw. Vorpuppen) zu erklären ist, oder im Zufallsbereich liegt, sei dahingestellt. Auf jeden Fall wurde bei den Versuchen darauf geachtet, daß die angebotenen Brut-Futtermengen gewichtsmäßig ausgeglichen waren (s.v). 


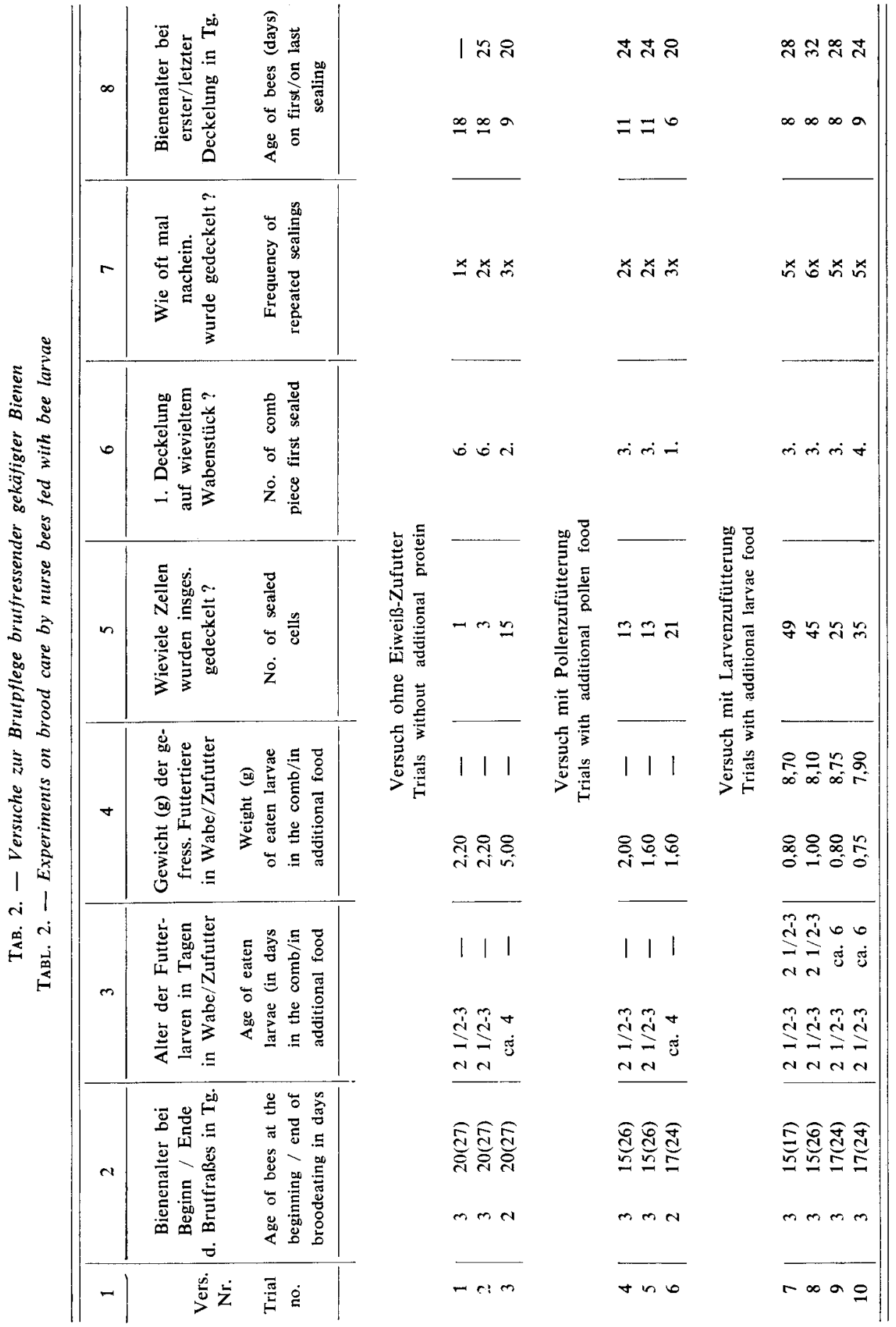


Die erste Deckelung erfolgte bei diesen Versuchen auf dem 3. bzw. 4. der nacheinander gebotenen Wabenstücke, nachdem die Pflegebienen 8 bzw. 9 Tage alt geworden waren. Danach kam es noch zu 4 bzw. 5 weiteren Deckelungen. Die letzten gedeckelten Zellen erschienen, als die Pflegebienen bereits zwischen 24 und 32 Tage alt waren. Die. Bienen waren somit längst dem normalen Brutpflegealter (3.-10. Lebenstag) entwachsen und befanden sich schon 2-3 Wochen im Sammelbienenalter, als sie immer noch Brut aufzogen.

3. Alle Versuche zusammengenommen stellen wir fest, daß die Versuchsbienen die Larven aus den Wabenstücken und den täglich neu gefüllten Schälchen bis zu ihrem 15. (bzw. 17. und 20.) Lebenstag rasch und vollständig verzehrten. Danach blieb von Mal zu Mal mehr Brutfutter übrig. Die Brut verfärbte sich dunkel und trocknete ein. Geringe Mengen sowohl von der im Schälchen gebotenen Brutnahrung, sowie von der Brut in den Wabenzellen haben die Bienen aber offenbar während der gesamten Zeit der Brutpflege gefressen.

4. Soweit die Bienen Zellen deckelten, waren es stets Arbeiterbrutzellen, niemals entstand eine Weiselzelle oder auch nur eine Andeutung dazu.

5. Um den 25. Versuchstag erschienen in den Kästchen mit zusätzlicher Pollennahrung die ersten Afterweiseleier in den ausgeräumten Zellen der Wabenstücke. In den Versuchskästchen ohne Pollen konnte ich bis zuletzt kein Ei entdecken.

6. Aus den gedeckelten Zellen schlüpften Bienen, welche zwar relativ klein, aber völlig normal gestaltet waren - gleich, ob sie von den nur brutfressenden oder den brut- und pollenverzehrenden Bienen aufgezogen wurden. Ich habe die unter den verschiedenen Fütterungsbedingungen aufgezogenen Bienen auch noch auf ihren physiologischen Zustand untersucht. Dazu wurden Ovarien, Fettkörper und Futtersaftdrüsen freipräpariert und einer mikroskopischen Beurteilung unterzogen. Technisch folgte ich dabei im wesentlichen den Angaben von MaURIzIO (1954), und hielt mich auch bei der Einordnung der Organe in einzelne Entwicklungsstufen an die von Maurizio gegebenen Beschreibungshilfen. Ich verwendete allerdings für jede der drei Kriterien eine Skala mit fünf Klassen, wobei das Stadium I die jeweils niedrigste und das Stadium V die höchste Entwicklungsstufe anzeigte. Die Übersicht in Tabelle 3 bekundet eine sehr ähnliche Klassenverteilung der untersuchten Merkmale der Einzelbienen in den einzelnen Versuchsgruppen und läßt auch bei den Klassemittelwerten keine spezielle Tendenz der Versuchsgruppen erkennen. Zwischen dem Nachwuchs der nur mit Brut und der mit Brut und Pollen ernährten Bienen bestanden keine merklichen physiologischen Unterschiede.

\section{DISKUSSION DER ERGEBNISSE}

Wenn die Bienen im Käfigversuch offen ausgelegte Brut fressen, kann man sich fragen, ob sie es aufgrund ihres Bedürfnisses nach dieser Kost oder im Zusammenhang mit ihrem angeborenen Reinigungstrieb tun. Dieser Trieb, der besonders im Stockbienenalter auftritt, ist bekanntlich auch im Käfigversuch wirksam. Er könnte zur Entfernung der unnatürlich umherliegenden Brutstadien reizen, wobei diese, einem Verhaltensinstinkt der Bienen zufolge, aufgefressen würden. Wenn man sah, wie die Bienen die Maden durch die Kästchen schleppten, an den Wänden hochzerrten, fallen ließen und wieder aufnahmen, mag man in dieser Ansicht bestärkt werden. Auch daß die Bienen trotz ausreichender Pollenversorgung nicht vom Brutfraß abließen, spricht nicht gegen die Mitwirkung des Säuberungsinstinktes beim Brutfraß.

Der merklich stärkere Brutverzehr der pollenlos gehaltenen Bienen deutet indessen bereits an, daß auch die Ernährungskomponente dabei von Bedeutung 


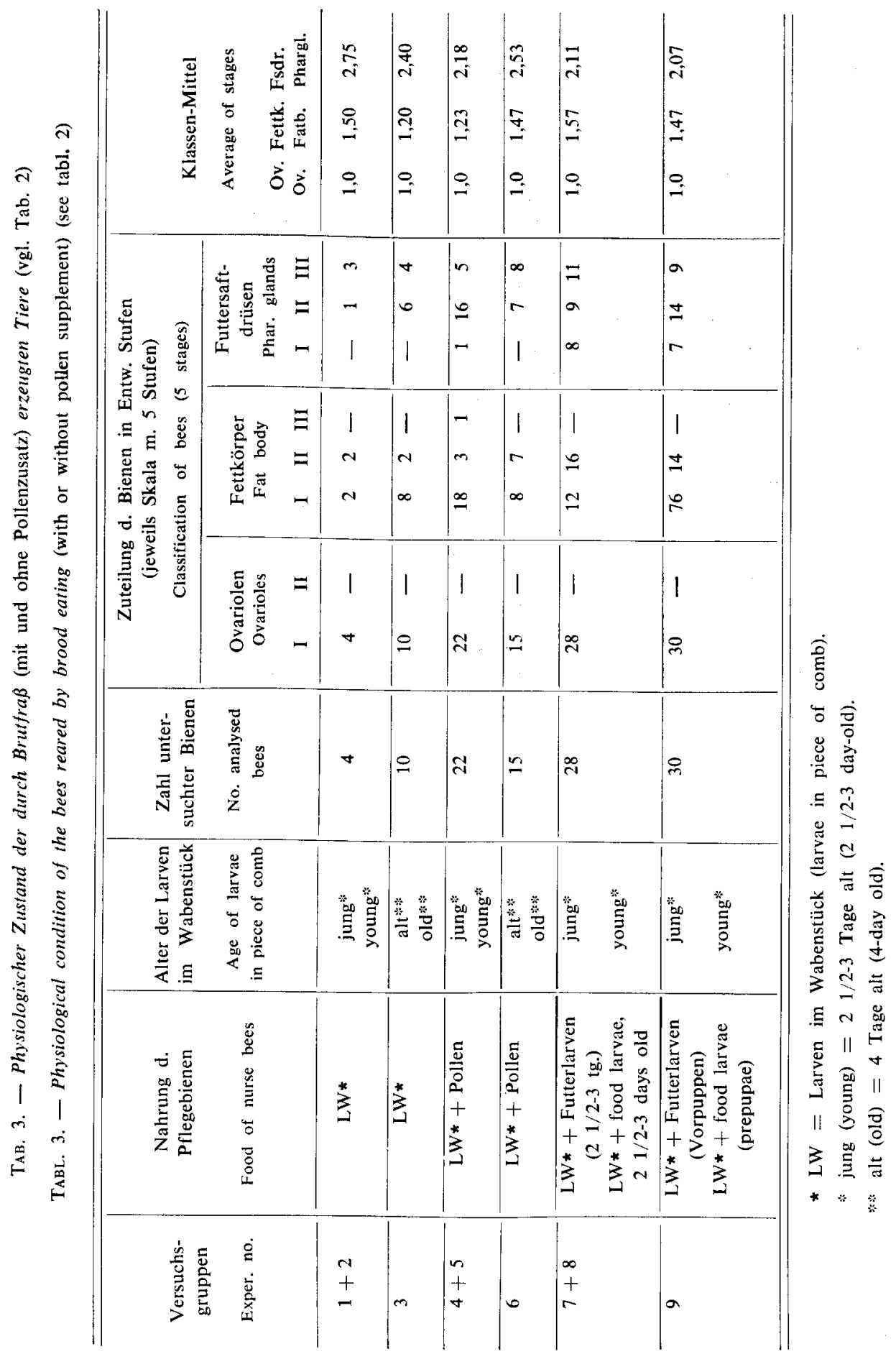


sein muß. Darauf weisen schließlich sehr eindeutig die Lebensdauerwerte der Bienen hin. Bienen, die Brut erhielten und fraßen, hatten dieselbe Lebenserwartung wie mit Pollen versorgte Bienen. Ohne Brut und Pollen lebten die Bienen bedeutend kürzer. Die Bienenbrut stellt also, zumindest hinsichtlich der Lebenserwartung der Bienen, einen vollen Ersatz des Pollens dar. Wenn die Lebensdauer der Bienen, an die sehr junge (3tägige) Brut und kein Pollen verfüttert wurde, etwas verkürzt erschien (Tab. 1, Vers. Nr. 7) — sie war immerhin etwas länger als bei den ohne Pollen und Brutfutter gehaltenen Bienen (Vers. Nr. 12) - so mag das einfach damit zusammenhängen, daß die gebotene Brutmenge in der Zeit des hohen Eiweißbedarfes der Bienen nicht ausreichte. Dies bestätigte schließlich auch der zweite Teil der Untersuchungen.

Bienen, die anstelle von Pollen mit Brut ernährt wurden, konnten Brut aufziehen. Wenn die Bienen ihren Eiweißbedarf aus Brutwaben stillen mußten, die ihnen gleichzeitig zur Brutpflege zu dienen hatten (Tab. 2, Vers. Nr. 1-3), war das Deckelungsergebnis mager. Das lag daran, daß die Menge des notwendigen Eiweißfutters in Form von überschüssigen Maden in den Zellen zur Brutpflege nicht ausreichte. Als die Bienen zusätzlich Pollen erhielten, war der Deckelungserfolg größer (Vers. Nr. 4-6). Am meisten Brut pflegten die gekäfigten Bienen jedoch, wenn ich ihnen anstelle von Pollen Brut in Schälchen reichte (Vers. Nr. 7-10). Über den Grund für diesen noch besseren Effekt der brutfressenden Bienen möchte ich hier nicht spekulieren. Bei allen Versuchen pflegten die Bienen noch Brut, als sie schon längst aus dem Ammenalter hinausgewachsen waren.

Die Versuche zeigten einmal mehr, daß die Pollenkomponente für die Erbruitung fertiger Bienen nicht unbedingt notwendig ist. Futtersaft und Zuckerfutter genügen. Dafür gibt es bereits eine Reihe von Hinweisen in der Bienenliteratur (z.B. Himmer, 1927 ; Whitcomb u. Wilson, 1927 ; Hydak, 1935 ; BuHCNER, 1953). Die hier dargestellten Versuche machen deutlich, daß die Bienen Futtersaft nicht nur aus Pollen, sondern auch durch den Verzehr von Bienenbrut erzeugen können und damit in der Lage sind, Brut aufzuziehen.

Die Fähigkeit der Brutaufzucht durch Brutfraß könnte eine Art Schutzeinrichtung für das Bienenvolk darstellen. Wenn im Laufe des Jahres, insbesondere während der Aufwärtsentwicklung der Biencnvölker, wetterbedingter Pollenmangel eintritt, kann der Brutfra $\beta$ der Biene verhindern, daß funktionsuntüchtige Individuen aufgezogen werden bzw. die angepflegte Brut zur Gänze ausgeräumt werden muß. Durch die Reduzierung der Brut und der Pflege eines Restbestandes mit Hilfe des Eiweißgewinnes aus der verzehrten Brut sind die Bienen fähig, Notzeiten leichter zu überstehen.

Man könnte hier an die eigenartige Erscheinung der Speichelabgabe bei den Wespenlarven (Gattung Paravespula und Vespa) an die erwachsenen Nestgenossen 
erinnert werden, worin MASCHWITZ (1966) eine Art Nahrungsausgleich im Wespenvolk sehen möchte. Er konnte zeigen, daß gekäfigte Wespen bei Anwesenheit von Wespenbrut mehrere Tage länger leben als ohne Brut. Diese Wirkung ist dem Zuckeranteil des Larvenspeichels (bei Vespa vulgaris im Durchschnitt $9 \%$ zu verdanken. Für die nicht Vorratswirtschaft treibenden Wespen kann damit in der freien Natur während Mangel- und Schlechtwetterzeiten eine echte Hilfe zum Überleben verbunden sein. Die Honigbiene hat es infolge ihrer Vorratswirtschaft nicht nötig, die Larven in den gegenseitigen Nahrungsaustausch einzubeziehen. Sie ist aber - ganz unpassend zu ihrer sonst rein vegetarischen Lebensweise in Notzeiten zu einer anderen regulativen Verhaltensweise in der Lage : sie verlängert ihr Leben und erhält sich die Fähigkeit zur Brutpflege durch den Verzehr von Brut, die sie aus natürlichen Nahrungsquellen nicht mehr versorgen kann. Wenn man will, kann man in den beiden unterschiedlichen Reaktionsweisen bei Wespen und Bienen ein Regulationsprinzip von allgemeiner Bedeutung sehen.

Eingegangen im Februar 1984.

Eingenommen im April 1984.

\section{DANKSAGUNG}

Bei der technischen Abwicklung der Versuche halfen mir Frau U. Bosch und Herr R. Riedel, wofür ich mich bedanke.

\section{RÉSUMÉ \\ REGULATION DU BILAN PROTÉINIQUE DANS UNE COLONIE D'ABEILLES (APIS MELLIFICA L.) PAR LA CONSOMMATION DE COUVAIN}

Dans la pratique apicole, aussi bien qu'en recherche apidologique, de nombreuses observations ont été faites concernant du couvain (œufs et larves) qui disparaît de la colonie sans avoir effectué son cycle de développement. Une question en découle : le cannibalisme du couvain a-t-il une signification biologique pour les abeilles?

I. Des abeilles récemment écloses ont été mises dans des cagettes en bois, à raison de 50 par cagette, et nourries avec une solution de saccharose à $50 \%$. En plus elles ont reçu dans des coupelles du couvain d’âge variable (larves, prénymphes nymphes). On a fourni également du pollen à une partie seulement des cagettes. Les abeilles d'une cage ont reçu du sirop et du pollen uniquement, et celles d'une autre cage que du sirop. Durant les expériences les cagettes ont été placées dans une étuve $\left(30^{\circ} \mathrm{C}, 60 \% \mathrm{HR}\right)$.

A partir du $3^{r}$ jour les abeilles ont commencé à manger le couvain et - tant qu'on leur en a fourni - le pollen. Elles ont consommé le pollen jusqu'au $12^{*}$ jour et le couvain jusqu'au $25^{e}$ jour, mais en moindre quantité à partir du $15^{\mathrm{e}}$ jour. Les abeilles qui mangeaient du couvain étaient nerveuses et agressives et consommaient comparativement plus de sirop.

Les abeilles pourvues en pollen aussi bien que celles sans pollen ont mangé du couvain (Tabl. 1, essais no 1-6 et 7-11). Les 2 groupes ont eu une durée de vie égale, entre 33 et 46 jours. 
Les abeilles nourries avec de très jeunes larves et sans pollen ont vécu moins longtemps (essais $\mathrm{n}^{\circ} 7$ et 8 ). La raison en est une quantité de protéines insuffisante. Ce sont les abeilles témoins, nourries uniquement avec du sirop (essai $n^{\circ} 12$ ), qui ont vécu le moins longtemps (20 jours).

II. Dans une autre série d'expériences faites dans les mêmes conditions, chaque cage a reçu de façon continue des morceaux de rayons de couvain contenant des larves âgées de 2 jours $1 / 2$ à 3 jours. En plus de cela, un groupe ne reçut que du sirop, un autre du sirop et du pollen et un $3^{\circ}$ du sirop et du couvain dans des coupelles (larves âgées de 2 jours $1 / 2$ à 3 jours ou de 6 jours environ).

Les abeilles encagées ont pu élever du couvain grâce aux protéines apportées par la consommation de couvain. Elles n'ont pris soin que de quelques cellules (1-15 par cage) si elles ne recevaient que des morceaux de rayon de couvain continuellement renouvelés (Tabl. 2, essais $n^{0}$ 1-3). Un bien plus grand nombre de cellules a été operculé, si les abeilles recevaient des larves supplémentaires (essais $n^{0} 7-10$ ). Si elles recevaient du pollen à la place des larves, le résultat de l'élevage était un peu moins bon (essais $n^{\circ} 4-6$ ).

La consommation de couvain a duré jusqu'au $27^{e}$ jour et uniquement tant que les abeilles ont élevé du couvain. Les abeilles élevées par des ouvrières qui mangeaient du couvain étaient relativement petites, mais leurs organes externes et internes (ovaires, corps gras, glandes pharyngiennes) étaient développés normalement.

La capacité des abeilles encagées d'atteindre une longévité normale et d'élever du couvain en en mangeant pourrait offrir une chance de survie aux colonies, lorsqu'il y a un déficit en pollen et pourrait être interprétée comme un moyen d'auto-protection.

\section{SUMMARY}

\section{REGULATION OF THE PROTEIN BALANCE IN THE BEE COLONY (APIS MELLIFERA L.) BY BROOD CANNLBALISM}

In practical beekeeping and apicultural research many observations have been made of brood (eggs and larvae) from bee colonies without completing development. From this the question was derived : Is there a biological significance of brood cannibalism ?

I. Newly emerged bees were set into small wooden test-cages (50 bees per cage) and were fed with a solution of saccharose and water $(50 \%)$. In addition to this they also received brood of different ages in small dishes (larvae prepupae and pupae). One part of the cages was provided also with pollen. The bees of one cage received only sugar water and pollen and in another cage they got nothing but sugar water. For the experiments the bees were kept in an incubator $\left(30^{\circ} \mathrm{C}, 60 \%\right.$ r.h.).

The bees began to eat brood and - as long as it was offered - pollen. They ate pollen up to the 12th day and brood up to the $25 \mathrm{th}$ day, although to a minor extent after the 15th day of life. The brood-eating bees were nervous and aggressive and consumed comparatively more sugar water.

Bees provided with pollen as well as those without pollen ate brood (Tab. 1, trial-no. 1-6 and 7-11). Both groups had an equal lifespan between 33 and 46 days (to $50 \%$ mortality). Bees fed with very young larvae without pollen had shorter lifespans (trial-no. 7 and 8 ). The reason for this may have been insufficient quantity of protein. The shortest lifespan ( 20 days) occurred in control bees fed with sugar water only (trial-no. 12).

11. In another series of experiments under the same conditions each cage was provided continuously with pieces of broodcombs containing $21 / 2-3$ day-old larvae. In addition to this one 
group received only sugar water, another one sugar water and pollen and a third group sugar water and brood in small dishes (2,5-3 resp. ca. 6 day-old larvae).

With the protein consumed by brood-eating the caged bees were able to raise brood. They took care of only a few cells (1-15 per cage) if they received only the continuously renewed pieces of broodcomb in their cages (tab. 2, trial-no. 1-3). Considerably more cells were sealed if the bees obtained additional larvae (tiral-no. 7-10). If they got pollen instead of larvae the result of broodrearing was a little worse (trial no. 4-6).

Brood-eating lasted until the 27 th day of life and only as long as becs reared brood. The bees reared by brood-eating bees were relatively small but their exterior and their inner organs (ovaries, fat body, pharyngeal glands) were developed normally.

The ability of caged bees to reach the normal longevity and to rear some brood by eating brood could provide in natural conditions a chance for the survival of colonies when there is a lack of pollen and could be interpreted as a matter of self-protection.

\section{VERÖFFENTLICHUNGEN}

Böttcher F.K., Hirschfelder H., Weiss K., 1963. - Die Tätigkeit der Bayerischen Landesanstalt für Bienenzucht Erlangen im Jahre 1962. Imkerfreund, 18 (3), 74-80.

Buchner R., 1953. - Beeinflussung der Größe der Arbeitsbiene durch Raum- und Nahrungsmangel während der Larvenzeit. Roux'Arch. Entwicklungsmech., 146, 544-579.

Fukuda H., Sakagami S.E., 1968. - Worker brood survival in honeybees. Res. Popul. Ecol., 10 (1), 31-39.

Garófalo C.A., 1977. - Brood viability in normal colonies of Apis mellifera. J. apic. Res., 16 (1), $3-13$.

HaYdaK M.H., 1935. - Brood rearing by honeybee confined to a pure carbohydrate diet. J. econ. Entomol., 28, 657-660.

Himmer A., 1927. - Fortschritte auf dem Gebiet der Bienenkunde und Bienenzucht. Erl. Jahrb., $5,70-71$.

Maschwitz U., 1966. - Das Speichelsekret der Wespenlarven und seine biologische Bedeutung. Z. vergl. Physiol., 53, 228-252.

Maurizıo A., 1954. - Pollenernährung und Lebensvorgänge bei der Honigbiene (Apis mellifica L.). Landw. Jahrb. der Schweiz, 68 (2), 15-182.

Merrill J.H., 1924. - Scaled and unsealed brood. Am. Bee. J., 64 (9), 424-425.

MERz R., 1979. - Beobachtungen zum Verschwinden von Brut im Winter und frühen Frühling. Schweiz. Bienenztg., 102 (4), 168-169.

Montagner H., 1962. - Essais préliminaires de mesure de la capacité d'élevage dans la ruche. Ann. Abeille, 5 (3), 233-246.

MYser W.C., 1952. - Ingestion of eggs by honey bee workers. Am. Bee J., 92 (2), 67.

Nauleau G., 1960. - Sur les conséquences de diverses perturbations apportées au couvain dans la ruche : La désoperculation - les transpositions. Insectes soc., 7 (4), 333-343.

Newton D.C., Michl D.J., 1974. - Cannibalism as an indication of pollen insufficiency in honeybees : ingestion or recapping of manually-exposed pupae. J. apic. Res., 13 (4), 235-241. 
Schulz-Langner E., 1956. - Ein neues Bild der bösartigen Faulbrut. Z. Bienenforsch., 3 (7), 149-180.

Weiss K., 1962. - Untersuchungen über Drohnenerzeugung im Bienenvolk. Arch. Bienenkd., 39 (1), 1-7.

WeIss K., 1958. - Frühzeitig Zuchtdrohnen! Imkerfreund, 13 (9), 265-266.

Weiss K., 1969. - Ausgewähltes über Drohnen. Allg. dtsch. Imkerztg., (4, 5, 6), 100-106, 131-135, 164-166.

Wille H., Gerig L., 1976. - Massenwechsel des Bienenvolkes. IV. Zusammenspiel der Eilegetätigkeit der Königin, der Bienenschlüpfrate und der Lebensdauer der Arbeiterinnen (Apis mellifica L.). Schweiz. Bienenztg., 112 (1, 3, 5). 16-25 125-140 244-257.

Whiтcoms W.W., WiLson H.F., 1927. - A suggested explenation of why bees appear to use pollen substitutes for brood rearing. Am. Honey Producer, 1 (3), 36-38.

Woyke J., 1963. - What happens to diploid drone larvae in a honeybee colony. J. apic. Res., 2 (2), 73-84.

WoYke J., 1976. - Brood-rearing efficiency and absconding in Indian honeybees. J. apic. Res., $15(3 / 4), 133-143$.

WOYKe J., 1977. - Cannibalism and Brood-rearing efficiency in the honeybee. J. apic. Res., 16 (1), 84-94. 